QUARTERLY OF APPLIED MATHEMATICS

VOLUME LXV, NUMBER 2

JUNE 2007, PAGES 385-402

$\mathrm{S} 0033-569 \mathrm{X}(07) 01049-\mathrm{X}$

Article electronically published on April 10, 2007

\title{
ON END ROTATION FOR OPEN RODS UNDERGOING LARGE DEFORMATIONS
}

\author{
$\mathrm{BY}$ \\ G. H. M. VAN DER HEIJDEN (Centre for Nonlinear Dynamics and its Applications, University \\ College London, Gower Street, London WC1E 6BT), \\ M. A. PELETIER (Technische Universiteit Eindhoven, Den Dolech 2, P.O. Box 513, 5600 MB \\ Eindhoven), \\ AND \\ R. PLANQUÉ (Department of Mathematics, Faculty of Sciences, Vrije Universiteit, De Boelelaan \\ 1081a, $1081 \mathrm{HV}$ Amsterdam)

\begin{abstract}
We give a careful discussion of end rotation in elastic rods, focusing on ambiguities that arise if arbitrarily large deformations are allowed. By introducing a closure and restricting to a class of deformations we show that a rigorous treatment of end rotation can be obtained. The results underpin various non-rigorous discussions in the literature and serve to promote the variational analysis of boundary-value problems for rods undergoing large deformations. As an example we discuss the application to the model of a rod lying on the surface of a cylinder.
\end{abstract}

\section{Introduction.}

1.1. Problem setting. The use of variational methods in the study of elastic bodies in equilibrium - thin elastic rods, in the context of this paper - is only possible if the external (prescribed) loads are conservative, i.e., such that the work done by that load along a path in state (conformation) space is independent of the path, and depends only on the initial and final states. Implicitly such a load defines a work functional on the state space, of which the load itself can be interpreted as a derivative.

In this context the case of elastic rods, loaded by their ends, is a particularly interesting one. Alexander \& Antman showed in a seminal paper both (1) that a moment, applied

Received August 28, 2006.

2000 Mathematics Subject Classification. Primary 49N99, 51H99, 51N99, 74B20, 74K10.

Key words and phrases. Rod theory, end rotation, twist, writhe, large deformations.

This research was partly funded by the EPSRC under contract GR/R51698/01. The first author also thanks The Royal Society for continuing support. The authors are grateful to Jason Cantarella for many interesting discussions, and to Rubber Import Amsterdam BV for supplying us with ample experimental material.

E-mail address: rplanque@few.vu.nl 
(a)

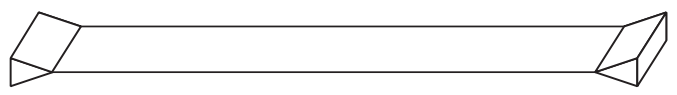

(b)

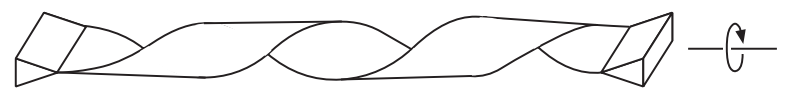

(c)

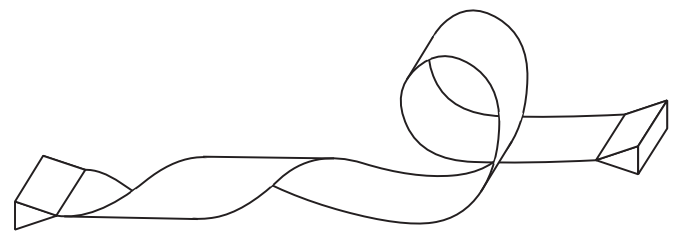

(d)

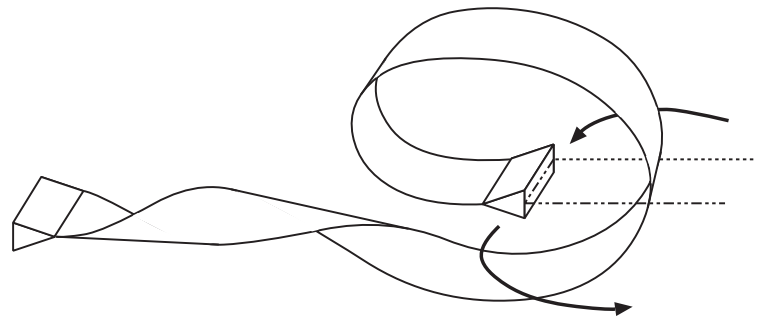

FIG. 1. The sequence (a)-(b)-(c)-(d)-(a) is a closed path in conformation space with non-zero end rotation. As a result, even a constant end moment is non-conservative. (After [2].)

to the end of a rod, is in general not conservative, and (2) that this can be remedied by suitably restricting the class of admissible deformations of the rod [2].

The fact that a moment applied at the end of a rod (or, to be more precise, a pair of equal and opposite moments applied to both ends) is not conservative is easily shown by an example. If we rotate the right end of the rod in Figure 1(a) through an angle of $-4 \pi$ we obtain the conformation shown in (b). If we now move the right end of the rod to the left, the rod pops into a looped conformation as shown in (c). If we move the right end further and make the loop pass around the right clamp, as in (d), and then pull the ends out again, we return to conformation (a) without having further rotated the ends. (Note that the process illustrated in Figure 1(d) requires that whatever supports the right clamp must be released to allow the passage of the rod behind it.) During this sequence, which starts and ends with conformation (a), one end remains fixed, and the other rotates about a fixed centerline tangent; an applied moment will therefore have done non-zero work along such a path. This shows that an applied moment may not be conservative. Note that the example only shows a lack of conservation in the case of a large closed path in conformation space. Indeed, Alexander \& Antman showed (and arguments below will reconfirm) that for moderate perturbations of the conformation an end moment is conservative. We thus have local conservation, but lack of conservation for large perturbations. 
This example also illustrates that the lack of conservation is intimately related to a different question concerning end rotation. End rotation is defined for a deformation process, a path in conformation space, along which the centerline tangents at the ends remain fixed. The end rotation $R$ associated with this path is then the relative rotation of the ends about their respective tangents (see Definition 2.11). The work done by a constant, non-zero pair of end moments of magnitude $M$ along this path equals $M R$. For this pair of moments to be conservative it is clearly necessary and sufficient that the end rotation $R$ is determined completely by the initial and final conformations and is independent of the connecting path. Therefore we now shift our focus, moving away from the issue of conservative end moments, towards the question whether end rotation is conservative. This will be the issue that we focus on in the rest of the paper.

1.2. The closure. Alexander \& Antman proposed a way to deal with the lack of global conservation. Their solution is to assume the elastic rod to be part of a longer rod that itself is closed (i.e., the centerline is a closed curve). For a closed rod the relative end rotation - the total rotational shear between the two ends, which are now adjacent - is known as the link (see (44)). For integer values the link is the topological linking number of two closed curves, one being the rod's centerline and the other a line drawn parallel to it on the surface of the unstressed rod; a straightforward extension exists to fractional values [7. The link is a topological invariant: once the ends are glued together the link is preserved no matter how the rod is deformed, provided no self-intersections of the rod are allowed.

The assumption that the rod under consideration is part of a larger, closed rod allowed Alexander \& Antman to define a class of deformations for which end moments are conservative. If we keep the virtual part of the closed rod (which we shall call the closure) fixed during deformation, variations in link can be interpreted as variations in end rotation of the rod. Provided the physical part of the rod (which we shall refer to as the 'open rod') does not intersect itself or the closure during deformation, the relative end rotation thus defined depends only on the initial and final states; with respect to this class of deformation an applied moment thus becomes conservative.

The class of allowable deformations now contains an element of arbitrariness: the open rod should not intersect the closure, but the closure itself is not part of the original setup; it was invented to make the variational formulation possible. However, as Alexander \& Antman remark, one may view the closure as the mathematical counterpart of the experimental apparatus that is used to control the rod ends; indeed, in a typical experimental setup the sequence of Figure 1 would be impossible without releasing the rod from the clamp that holds it.

1.3. Moving supports. For fixed closures - implying fixed end positions - this approach provides a satisfactory framework for a variational analysis. For moving supports, however, the closure will have to change during deformation, and then the situation is less clear. If no constraints are placed on the deformation of the closure, then the example of Figure 1 is again allowed, for the closure can simply change such as to accommodate the movement of the open rod. In addition, the degrees of freedom of a rod contain not only a measure of centerline position but also a measure of rotation about the centerline. It is a priori unclear how this rotation should be chosen when the closure changes. 
The main purpose of this paper, therefore, is to investigate the conditions that we should place on the closure and the admissible deformations in order to obtain a globally conservative end rotation. In Section 2 we introduce such conditions, in the form of a class of conformations that can be linked to a reference conformation by a deformation process satisfying certain requirements (the class $\mathcal{A}_{r_{0}}^{2}$, Definition 2.7). For deformations in this class the end rotation is indeed conservative: its value can be inferred from the reference and final conformations. If the reference conformation is straight, then in terms of an appropriate choice of Euler angles the end rotation is given by the simple formula (see Section 2.3)

$$
R=\int[\dot{\phi}+\dot{\psi}] d s .
$$

In Section 3 we illustrate the application of these results with the example of a rod constrained to lie on the surface of a cylinder.

The formula (11) is by no means new, and has been used extensively in the literature on elastic rods (often in applications to DNA supercoiling). Our contribution consists in making the assumptions explicit that are required for (11) to be meaningful, and in highlighting the pitfalls that surround this expression. Indeed, the class of admissible deformations $\mathcal{A}_{r_{0}}^{2}$ is an ugly beast, and in Section 4 we critically review the defining conditions of this class, using counterexamples to show that they are necessary.

Finally, in Section [5 we discuss our approach in light of other existing work.

\section{Results: Open twist, open writhe and end rotation.}

2.1. Setup. For our purposes a rod is a member of the set

$$
\begin{aligned}
& \mathcal{A}^{0}=\left\{\left(r, d_{1}\right) \in C^{2}\left([0, L] ; \mathbb{R}^{3} \times S^{2}\right) \text { such that }|\dot{r}| \neq 0, \dot{r} \cdot d_{1}=0,\right. \\
&\text { and } r \text { is non-self-intersecting }\} .
\end{aligned}
$$

Here and in the following an overdot denotes differentiation with respect to the spatial variable $s$. The curve $r$ is thought of as the centerline of a physical rod, and $d_{1}(s)$ as a material vector in the section at $s$. As alternatives to 'rod' the terms 'ribbon' [7, 1] and 'strip' 22 are also used. A closed rod is an element of $\mathcal{A}^{0}$ for which begin and end connect smoothly.

To each point on the centerline of the rod we can attach an orthonormal right-handed frame $\left(d_{1}(s), d_{2}(s), d_{3}(s)\right)$ of directors by setting

$$
d_{3}(s)=\dot{r}(s) /|\dot{r}(s)| \quad \text { and } \quad d_{2}(s)=d_{3}(s) \times d_{1}(s) .
$$

These directors track the varying orientation of the cross-section of the rod along the length of the rod. The twist of a closed rod $\left(r, d_{1}\right)$ is now defined by

$$
T w\left(r, d_{1}\right):=\frac{1}{2 \pi} \int_{0}^{L} \dot{d}_{1}(s) \cdot d_{2}(s) d s .
$$

It measures the number of times $d_{1}$ revolves around $d_{3}$ in the direction of $d_{2}$ as we go around the rod. 
Let $r_{1}$ and $r_{2}$ be two non-intersecting closed curves. Then the link of $r_{1}$ and $r_{2}$ is defined by

$$
L k\left(r_{1}, r_{2}\right):=\frac{1}{4 \pi} \int_{0}^{L} \int_{0}^{L} \frac{\left[\dot{r}_{1}(s) \times \dot{r}_{2}(t)\right] \cdot\left[r_{1}(s)-r_{2}(t)\right]}{\left|r_{1}(s)-r_{2}(t)\right|^{3}} d s d t .
$$

The writhe of a closed curve $r$ is

$$
W r(r):=\frac{1}{4 \pi} \int_{0}^{L} \int_{0}^{L} \frac{[\dot{r}(s) \times \dot{r}(t)] \cdot[r(s)-r(t)]}{|r(s)-r(t)|^{3}} d s d t .
$$

The argument of this integral is the pullback of the area form on $S^{2}$ under the Gauss map $\mathbb{R}^{2} \longrightarrow S^{2}$

$$
G:(r(s), r(t)) \longmapsto \frac{r(s)-r(t)}{|r(s)-r(t)|},
$$

so that the writhe may be interpreted as the signed area on $S^{2}$ that is covered by this map. For each direction $p \in S^{2}$ the signed multiplicity of the Gauss map (i.e., the number of points $(s, t)$ for which $G(s, t)=p$, weighted by the sign of $\left.p \cdot\left[G_{s} \times G_{t}\right]\right)$ equals the directional writhing number, the number of signed crossings of the projection of $r$ onto a plane orthogonal to the vector $p$ [6, 1]. In other words, the writhe of a closed curve is equal to the directional writhing number averaged over all directions of $S^{2}$.

The link, twist and writhe of a closed rod are related by the well-known Călugăreanu-White-Fuller Theorem 4, 20, 6]:

Theorem 2.1. Let $\left(r, d_{1}\right) \in \mathcal{A}^{0}$ be a closed rod as defined above. Then

$$
L k\left(r, d_{1}\right)=T w\left(r, d_{1}\right)+W r(r) .
$$

We review two classical theorems by Fuller [7] which are of interest to us. Note that at each point $r(s)$, the unit tangent $t(s)=\dot{r}(s) /|\dot{r}(s)|$ traces out a closed curve on $S^{2}$, called the tantrix. Fuller's first theorem relates the writhe of the curve to the area $A$ enclosed by the tantrix on $S^{2}$ :

$$
W r(r)=\frac{A}{2 \pi}-1(\bmod 2) .
$$

Note that the equality modulo two is necessary since the area enclosed by a curve on $S^{2}$ is only defined modulo $4 \pi$.

The second theorem, stated in detail as Theorem 2.5 below, gives under certain conditions a formula for the difference in writhe between two closed curves $r_{1}$ and $r_{2}$ that can be continuously deformed into each other (see Figure 2):

$$
W r\left(r_{1}\right)-W r\left(r_{2}\right)=\frac{1}{2 \pi} \int \frac{t_{2} \times t_{1}}{1+t_{1} \cdot t_{2}} \cdot\left(\dot{t}_{1}+\dot{t}_{2}\right) .
$$

2.2. The set of admissible homotopies. We now proceed with the introduction of the set for which the end rotation (and twist and writhe functionals) will be defined. Let us first briefly sketch the underlying ideas:

(1) We close the rod with a closure as in 2] in order to prevent the situation of Figure 1 . 


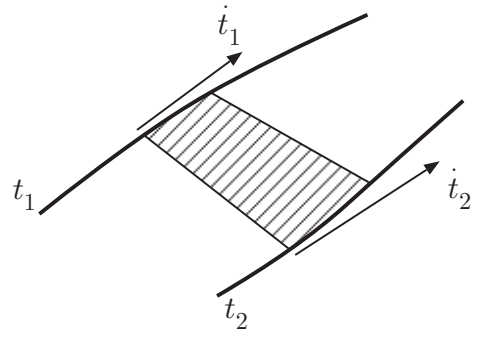

FIG. 2. The integral in (8) represents the area swept out by the geodesic connecting the curves $t_{1}$ and $t_{2}$ on $S^{2}$.

(2) In contrast to [2], the closure is forced to follow changes in the end points of the rod. We therefore have to consider homotopies of the rod with its closure, i.e., homotopies of closed rods.

(3) To fix the set of admissible homotopies we choose a fixed starting point, a closed planar curve $r_{0} \in C^{2}\left([0, M] ; \mathbb{R}^{3}\right)$ for some $M>L$, representing the initial rod with its closure.

Recall that the dot always denotes differentiation with respect to the parameter $s$.

DEFINITION 2.2.

$$
\mathcal{A}_{r_{0}}^{1}=\left\{\quad\left(r, d_{1}\right) \in \mathcal{A}^{0} \text { such that } \exists\left(\bar{r}, \bar{d}_{1}\right) \in C^{2}\left([0, M] \times[0,1] ; \mathbb{R}^{3} \times S^{2}\right):\right.
$$

1. for each $\lambda, \bar{r}(\cdot, \lambda)$ is an unknotted, non-self-intersecting closed curve,

2. $|\dot{\bar{r}}(s, \lambda)| \neq 0$ and $\dot{\bar{r}}(s, \lambda) \cdot \bar{d}_{1}(s, \lambda)=0$ for $s \in[0, M], \lambda \in\{0,1\}$,

3. $\left(\bar{r}, \bar{d}_{1}\right)(s, 1)=\left(r, d_{1}\right)(s)$ for $s \in[0, L]$,

4. $\bar{r}(s, 0)=r_{0}(s)$ for $s \in[0, M]$,

5. $\frac{\dot{\bar{r}}(s, 0)}{|\dot{\bar{r}}(s, 0)|} \cdot \frac{\dot{\bar{r}}(s, \lambda)}{|\dot{\bar{r}}(s, \lambda)|}>-1 \quad$ for $s \in[0, M], \lambda \in[0,1]$,

6. $\{\bar{r}(s, \lambda): s \in[L, M]\}$ is a planar curve for $\lambda=0$ and for $\lambda=1$, and the two planes are parallel $\}$.

$\mathcal{A}_{r_{0}}^{1}$ can be thought of as a class of open rods $\left(r, d_{1}\right)$ that can be first closed and then connected by a homotopy to the reference curve $r_{0}$. Both the closure and the homotopy are to satisfy certain requirements. The closure is the part of the closed rod corresponding to $s \in[L, M]$.

Some of the conditions above are more natural than others. Parts 1 and 2 state that $\left(\bar{r}, \bar{d}_{1}\right)$ is a homotopy of well-behaved closed rods, and by parts 3 and 4 the homotopy contains the original open $\operatorname{rod}\left(r, d_{1}\right)$ at $\lambda=1$, and the reference curve at $\lambda=0$. Parts 5 and 6 contain the essential elements of this definition. Part 5 is the same non-opposition condition that appears in the statement of Fuller's theorem (Theorem 2.5) and is required for the conversion of the writhe to a single-integral expression. Part 5 may appear to be a technical restriction, but as we discuss in Section 4 the conservation of end rotation will not hold if we simply remove this condition. Part 6 , which states that the closure should 
be planar at the beginning and the end of the homotopy, is central to the construction. This condition is inspired by the fact that a planar closed curve has zero writhe, a fact verified immediately in (5); the effect of this condition is to eliminate any contribution of the closure to the writhe of the closed curve, as is exploited in the proof of Theorem 2.4 below.

Note that curves $r_{0}$ exist for which $\mathcal{A}_{r_{0}}^{1}$ is empty: if the three vectors $\dot{r}_{0}(0), \dot{r}_{0}(L)$, and $r_{0}(L)-r_{0}(0)$ are independent, then the open curve $r_{0}$ cannot be closed by a planar closure, so that the set of homotopies with planar closures that connect to $r_{0}$ is empty.

We are now in a position to define the new functionals 'open twist' and 'open writhe' for open rods.

Definition 2.3. Let $\left(r, d_{1}\right)$ be a $\operatorname{rod}$ in $\mathcal{A}_{r_{0}}^{1}$. Then the open twist of $\left(r, d_{1}\right)$ is

$$
T w^{o}\left(r, d_{1}\right):=\frac{1}{2 \pi} \int_{0}^{L} \dot{d}_{1} \cdot\left(\dot{r} \times d_{1}\right) d s,
$$

and the open writhe of $r$ is

$$
W r^{o}(r):=W r(\bar{r}(\cdot, 1))=\frac{1}{4 \pi} \int_{0}^{M} \int_{0}^{M} \frac{[\bar{r}(s, 1)-\bar{r}(t, 1)] \cdot[\dot{\bar{r}}(s, 1) \times \dot{\bar{r}}(t, 1)]}{|\bar{r}(s, 1)-\bar{r}(t, 1)|^{3}} d s d t .
$$

Theorem 2.4. For any open $\operatorname{rod}\left(r, d_{1}\right) \in \mathcal{A}_{r_{0}}^{1}$, the open twist and writhe are well-defined.

The definitions of open twist and twist for closed curves are identical, and the wellposedness of open twist is obvious. For writhe, however, different homotopy closures $\left(\bar{r}, \bar{d}_{1}\right)$ might give rise to different values of $W r^{\circ}(r)$, and a proof of well-posedness is required.

Proof. We first state Fuller's second theorem in a more precise form.

Theorem 2.5 ([7). Let $r_{\lambda}(0 \leq \lambda \leq 1)$ be a homotopy of closed non-self-intersecting curves, regularly parametrised with a common parameter $s \in[0, L]$. Let $t_{\lambda}=\dot{r}_{\lambda} /\left|\dot{r}_{\lambda}\right|$ be the tantrix of $r_{\lambda}$. If $t_{0}(s) \cdot t_{\lambda}(s)>-1$ for all $s \in[0, L], \lambda \in[0,1]$, then

$$
W r\left(r_{1}\right)-W r\left(r_{0}\right)=\frac{1}{2 \pi} \int_{0}^{L} \frac{t_{0} \times t_{1}}{1+t_{0} \cdot t_{1}} \cdot\left(\dot{t}_{0}+\dot{t}_{1}\right) d s .
$$

A detailed proof of Theorem 2.5 can be found in [1.

To prove Theorem 2.4 let $\left(\bar{r}, \bar{d}_{1}\right)$ be a homotopy associated to $\left(r, d_{1}\right)$. By definition, $\bar{r}(\cdot, 0)$ and $\bar{r}(\cdot, 1)$ are planar for $s \in[L, M]$. Let us denote the planes by $V_{0}$ and $V_{1}$; these are parallel by Definition 2.2.6. Let $V$ be the plane through the origin parallel to both.

We have defined the class of open rods $\mathcal{A}_{r_{0}}^{1}$ such that Theorem 2.5 can be applied. Denote the tantrices of $\bar{r}(\cdot, 0)$ and $\bar{r}(\cdot, 1)$ by $t_{0}$ and $t_{1}$ respectively. Then

$$
W r(\bar{r}(\cdot, 1))-W r(\bar{r}(\cdot, 0))=\frac{1}{2 \pi} \int_{0}^{M} \frac{t_{0}(s) \times t_{1}(s)}{1+t_{0}(s) \cdot t_{1}(s)} \cdot\left(\dot{t}_{0}(s)+\dot{t}_{1}(s)\right) d s .
$$


The argument of the integral vanishes for $s \in[L, M]$ : since $t_{0}(s), t_{1}(s) \in V$ for $s \in[L, M]$ we have $t_{0}(s) \times t_{1}(s) \perp V$ and $\dot{t}_{0}(s)+\dot{t}_{1}(s) \in V$ for $s \in[L, M]$. Hence

$$
\left[t_{0}(s) \times t_{1}(s)\right] \cdot\left[\dot{t}_{0}(s)+\dot{t}_{1}(s)\right]=0 .
$$

Moreover, since $\bar{r}(\cdot, 0)$ is planar, $\operatorname{Wr}(\bar{r}(\cdot, 0))=0$. We conclude that

$$
W r(\bar{r}(\cdot, 1))=\frac{1}{2 \pi} \int_{0}^{L} \frac{t_{0}(s) \times t_{1}(s)}{1+t_{0}(s) \cdot t_{1}(s)} \cdot\left(\dot{t}_{0}(s)+\dot{t}_{1}(s)\right) d s .
$$

Since this integral only depends on the reference curve and the open rod itself, and is otherwise independent of the choice of closure and homotopy, this proves the claim.

REMARK 2.6. A natural question to ask is whether the open twist and writhe thus defined reduce to their classical counterparts when an open rod is converted into a closed rod by lining up and connecting the ends. For writhe this is not the case, as we demonstrate in Section 4

2.3. Aligned end tangents. It is common in applications that the end tangents of the buckled rod are kept constant and equal during the deformation process, and for comparison with an end rotation we introduce this additional condition. Throughout this section we also assume that $\left.r_{0}\right|_{[0, L]}$ is straight, and without loss of generality we choose the director $d_{1}$ constant on the reference curve $\left.r_{0}\right|_{[0, L]}$ :

DEFINITION 2.7.

$$
\begin{aligned}
\mathcal{A}_{r_{0}}^{2}=\left\{\left(r, d_{1}\right) \in \mathcal{A}_{r_{0}}^{1}:\right. & \frac{\dot{r}_{0}(s)}{\left|\dot{r}_{0}(s)\right|}=v \in S^{2} \text { for all } s \in[0, L], \\
& \frac{\dot{\bar{r}}(0, \lambda)}{|\dot{\vec{r}}(0, \lambda)|}=\frac{\dot{\bar{r}}(L, \lambda)}{|\dot{\bar{r}}(L, \lambda)|}=\frac{\dot{\bar{r}}(M, \lambda)}{|\dot{\bar{r}}(M, \lambda)|}=v \text { for all } \lambda \in[0,1], \\
& \left.\bar{d}_{1}(s, 0)=\bar{d}_{1}(0,0) \text { for all } s \in[0, L]\right\} .
\end{aligned}
$$

The following formula is a direct consequence of Theorem 2.5

Corollary 2.8. Let $\left(r, d_{1}\right) \in \mathcal{A}_{r_{0}}^{2}$ and let $t$ be the tantrix of $r$. Then

$$
W r^{o}(r)=\frac{1}{2 \pi} \int_{0}^{L} \frac{v \times t(s)}{1+v \cdot t(s)} \cdot \dot{t}(s) d s .
$$

In the present case of a straight $\left.r_{0}\right|_{[0, L]}$ the dependence on $r_{0}$ of the open writhe of a given open rod takes a particularly simple form:

Theorem 2.9. Under the conditions of Corollary 2.8, let $\Omega=S^{2} \backslash\{-t(s): s \in[0, L]\}$. Then the function

$$
v \in S^{2} \longmapsto \frac{1}{2 \pi} \int_{0}^{L} \frac{v \times t(s)}{1+v \cdot t(s)} \cdot \dot{t}(s) d s
$$

is constant on connected components of $\Omega$.

The proof is given in the appendix. The interpretation of this theorem is as follows: if the end tangents are aligned, then the tantrix given by the rod (without closure) forms a closed curve on $S^{2}$. The integral above represents the spherical area enclosed by the curve, either to the left or to the right of the curve ( $c f$. (7)). When the vector $v$ crosses 
the set $\{-t(s): s \in[0, L]\}$ the geodesic connections between $v$ and $t(s)$ change direction, causing the integral to represent the area on the other side of the curve, and therefore causing the integral to jump by $4 \pi$.

To study the relationship between end rotation and open twist and writhe we introduce a particular choice of Euler angles for an open $\operatorname{rod}\left(r, d_{1}\right)$. Recall that for every $s \in[0, L]$ there is an orthonormal director frame $\left(d_{1}(s), d_{2}(s), d_{3}(s)\right)$. We express this frame in terms of angles $\theta, \psi, \phi$ with respect to a fixed basis $\left(e_{1}, e_{2}, e_{3}\right)$ as follows:

$$
\begin{aligned}
d_{1}= & (-\sin \psi \sin \phi+\cos \psi \cos \phi \cos \theta) e_{1} \\
& +(\cos \psi \sin \phi+\sin \psi \cos \phi \cos \theta) e_{2}-\cos \phi \sin \theta e_{3}, \\
d_{2}= & (-\sin \psi \cos \phi-\cos \psi \sin \phi \cos \theta) e_{1} \\
& +(\cos \psi \cos \phi-\sin \psi \sin \phi \cos \theta) e_{2}+\sin \phi \sin \theta e_{3}, \\
d_{3}= & \cos \psi \sin \theta e_{1}+\sin \psi \sin \theta e_{2}+\cos \theta e_{3} .
\end{aligned}
$$

This choice of Euler angles follows Love [9, art. 253]. For rods in the class $\mathcal{A}_{r_{0}}^{2}$ we choose $e_{3}$ parallel to $v$; note that by this choice the non-opposition condition 5 in Definition 2.2 coincides with avoidance of the Euler-angle singularity at $\theta=\pi$. Therefore the smoothness assumptions on $\left(\bar{r}, \bar{d}_{1}\right)$ in $\mathcal{A}_{r_{0}}^{2}$ imply $C^{1}$-regularity for $\phi, \psi$, and $\theta$.

In terms of these Euler angles the following formulae for open twist, open writhe and end rotation are obtained.

Lemma 2.10. Let $\left(r, d_{1}\right)$ be an open $\operatorname{rod}$ in $\mathcal{A}_{r_{0}}^{2}$. Then

$$
T w^{o}\left(r, d_{1}\right)=\frac{1}{2 \pi} \int_{0}^{L}[\dot{\phi}(s)+\dot{\psi}(s) \cos \theta(s)] d s
$$

and

$$
W r^{o}(r)=\frac{1}{2 \pi} \int_{0}^{L} \dot{\psi}(s)(1-\cos \theta(s)) d s
$$

Proof. The formula for twist is easily found by using (14) in the definition of open twist (Definition 2.3). For the writhe we apply Corollary 2.8 and use the fact that $v=e_{3}$.

With fixed end tangents we can introduce the end rotation. We denote $\partial(\cdot) / \partial \lambda$ by $\partial_{\lambda}(\cdot)$.

Definition 2.11. Let $\left(r, d_{1}\right) \in \mathcal{A}_{r_{0}}^{2}$ be an open rod with an associated homotopy $\left(\bar{r}, \bar{d}_{1}\right)$, and let $\bar{d}_{2}$ and $\bar{d}_{3}$ be constructed from $\bar{r}$ and $\bar{d}_{1}$ according to (2). We define the end rotation by

$$
R\left(r, d_{1}\right):=\int_{0}^{1} \partial_{\lambda} \bar{d}_{1}(L, \lambda) \cdot \bar{d}_{2}(L, \lambda) d \lambda-\int_{0}^{1} \partial_{\lambda} \bar{d}_{1}(0, \lambda) \cdot \bar{d}_{2}(0, \lambda) d \lambda .
$$

We can now prove the following. 
Lemma 2.12. With a slight abuse of notation, let $\phi, \psi, \theta:[0, M] \times[0,1] \rightarrow \mathbb{R}$ be the Euler-angle representation of $\left(\bar{d}_{1}, \bar{d}_{2}, \bar{d}_{3}\right)$. Then

$$
R\left(r, d_{1}\right)=\int_{0}^{L}[\dot{\phi}(s, 1)+\dot{\psi}(s, 1)] d s .
$$

From this lemma we conclude

Corollary 2.13. For open rods $\left(r, d_{1}\right) \in \mathcal{A}_{r_{0}}^{2}$,

$$
\frac{1}{2 \pi} R\left(r, d_{1}\right)=W r^{o}(r)+T w^{o}\left(r, d_{1}\right) .
$$

In particular, $R\left(r, d_{1}\right)$ is independent of the choice of extension $\left(\bar{r}, \bar{d}_{1}\right)$.

Proof of Lemma 2.12, Using the definitions of the Euler angles, we find

$$
\partial_{\lambda} \bar{d}_{1}(s, \lambda) \cdot \bar{d}_{2}(s, \lambda)=\partial_{\lambda} \phi(s, \lambda)+\partial_{\lambda} \psi(s, \lambda) \cos \theta(s, \lambda) .
$$

For $s=0, L$ and $\lambda \in[0,1]$ we have set $\bar{d}_{3}(s, \lambda)=e_{3}$, and hence $\theta(s, \lambda)=0$ for $s=0, L$; therefore $\partial_{\lambda} \bar{d}_{1}(s, \lambda) \cdot \bar{d}_{2}(s, \lambda)=\partial_{\lambda} \phi(s, \lambda)+\partial_{\lambda} \psi(s, \lambda)$ for $s=0, L$.

Since $\phi+\psi$ is a continuously differentiable function on $V:=[0, L] \times[0,1]$, the integral of the tangential derivative of $\phi+\psi$ along $\partial V$ vanishes:

$$
\oint_{\partial V} \frac{\partial}{\partial \tau}(\phi+\psi)=0
$$

where $\tau$ is the clockwise-pointing unit vector tangential to $\partial V$.

Hence

$$
\begin{aligned}
\int_{0}^{L}\left[\partial_{s} \phi\right. & \left.(s, 1)+\partial_{s} \psi(s, 1)\right] d s-\int_{0}^{L}\left[\partial_{s} \phi(s, 0)+\partial_{s} \psi(s, 0)\right] d s \\
= & \int_{0}^{1}\left[\partial_{\lambda} \phi(L, \lambda)+\partial_{\lambda} \psi(L, \lambda)\right] d \lambda-\int_{0}^{1}\left[\partial_{\lambda} \phi(0, \lambda)+\partial_{\lambda} \psi(0, \lambda)\right] d \lambda \\
= & \left.\int_{0}^{1}\left[\partial_{\lambda} \phi(s, \lambda)+\partial_{\lambda} \psi(s, \lambda)\right] d \lambda\right|_{s=0} ^{s=L} \\
= & \left.\int_{0}^{1} \partial_{\lambda} \bar{d}_{1}(s, \lambda) \cdot \bar{d}_{2}(s, \lambda) d \lambda\right|_{s=0} ^{s=L}=R\left(r, d_{1}\right) .
\end{aligned}
$$

3. Example: Rod on a cylinder. In [17, 18, the present authors studied the deformation of an inextensible and unshearable rod constrained to lie on a cylinder. The rod is assumed to be loaded by an axial end force (tension) $T$ and an end twisting moment $M$ both of which are applied along the axis of the cylinder, which we take parallel to $e_{3}$. The loading device is assumed to leave the end of the rod free to rotate around the circumference of the cylinder. Thus we have $d_{3}(0)=d_{3}(L)=e_{3}$, but the ends of the rod need not be coaxial. We shall now write down the total potential energy. This will involve the end rotation through which $M$ does work. We use the Euler angles $\theta, \psi, \phi$ defined in (14). The inextensibility/unshearability constraint $\dot{r}=d_{3}$, combined with the 
cylindrical constraint $\left(r \cdot e_{1}\right)^{2}+\left(r \cdot e_{2}\right)^{2}=a^{2}$, where $a$ is the radius of the cylinder, yields the kinematic condition

$$
\dot{\psi}=\frac{1}{a} \sin \theta
$$

The generalised strain vector $u=\kappa_{1} d_{1}+\kappa_{2} d_{2}+\tau d_{3}$ is defined by $\dot{d}_{i}=u \times d_{i} . \kappa_{1}$ and $\kappa_{2}$ are the curvatures about $d_{1}$ and $d_{2}$, respectively, while $\tau$ is the twist. Taking account of (18), we obtain

$$
\kappa_{1}=\dot{\theta} \sin \phi-\frac{1}{a} \sin ^{2} \theta \cos \phi, \quad \kappa_{2}=\dot{\theta} \cos \phi+\frac{1}{a} \sin ^{2} \theta \sin \phi, \quad \tau=\dot{\phi}+\frac{1}{a} \sin \theta \cos \theta .
$$

Note that because of inextensibility all derivatives are now with respect to arclength $s$.

The strain energy in the rod is taken to be the usual expression of linear elasticity

$$
E(\theta, \tau)=\frac{B}{2} \int_{0}^{L}\left(\kappa_{1}^{2}+\kappa_{2}^{2}\right)+\frac{C}{2} \int_{0}^{L} \tau^{2}=\frac{B}{2} \int_{0}^{L}\left(\dot{\theta}^{2}+\frac{1}{a^{2}} \sin ^{4} \theta\right)+\frac{C}{2} \int_{0}^{L} \tau^{2} .
$$

Here $B$ and $C$ are the bending and torsional stiffnesses of the (transversely isotropic) rod.

The displacement corresponding to the applied force $T$ is the end shortening $S$, for which we can write

$$
S=\int_{0}^{L}(1-\cos \theta)
$$

while the generalised displacement corresponding to the applied moment $M$ is the end rotation $R$ given by Definition 2.11. Provided an appropriate class of admissible deformations can be found, we can apply the preceding discussion to obtain

$$
R=\int_{0}^{L}(\dot{\phi}+\dot{\psi})=[\phi+\psi]_{0}^{L} .
$$

To construct our class of admissible deformations we choose as our reference conformation the straight rod $(\theta \equiv 0)$. This open rod can be made into a smooth planar closed structure (with centerline $r_{0}$ ) by means of an approximately semi-circular closure that can be chosen in any plane through the reference rod. Because the final rod conformation has aligned (although in general displaced) ends, there is always a plane in which the rod can be closed by means of a smooth closure (assuming one end tangent is kept fixed in the deformation), namely the plane through the two lines of the end tangents. This final plane is parallel to one of the infinity of planes of the initial closed conformation and therefore condition 6 of Definition 2.2 is satisfied. If we assume that $\theta$ remains bounded away from $\pm \pi$ - and note that we are forced to assume this, since we have no argument to support this claim - we also satisfy the non-opposition condition 5 of Definition 2.2 . We have thus verified that we have a class of deformations for which (22) gives the correct end rotation. 
The total potential energy is the sum of $E(\theta, \tau)$ and the work contributions of the external loads:

$$
\begin{aligned}
& V(\theta, \tau)=E(\theta, \tau)+T S-M R \\
& =\int_{0}^{L}\left[\frac{B}{2} \dot{\theta}^{2}+\frac{B}{2 a^{2}} \sin ^{4} \theta+\frac{C}{2} \tau^{2}+T(1-\cos \theta)-M\left(\tau+\frac{1}{a} \sin \theta(1-\cos \theta)\right)\right] .
\end{aligned}
$$

We can obtain a reduced minimisation problem by performing the minimisation with respect to $\tau$, which gives $\tau \equiv M / C$. Re-insertion then yields the final functional to be minimised:

$$
F(\theta)=\int_{0}^{L}\left[\frac{B}{2} \dot{\theta}^{2}+\frac{B}{2 a^{2}} \sin ^{4} \theta-T \cos \theta-\frac{M}{a} \sin \theta(1-\cos \theta)\right] .
$$

In [18] this functional, under slightly different loading conditions, is studied subject to the constraint that the rod cannot pass through itself on the cylinder.

4. Critique. The class $\mathcal{A}_{r_{0}}^{1}$ is arguably neither elegant nor practical. In addition, the explicit dependence on the deformation history is at odds with our intuition that stationary elastic problems should be formulated in terms of the current conformation only. Nonetheless, we claim two reasons for studying this approach to end rotation: first, it is a formalization of current literature practice, and second, there currently exists (to the best of our knowledge) no better way to treat moving supports. The first claim is elaborated in Section 5 in the current section we will show to which extent the definition of $\mathcal{A}_{r_{0}}^{1}$ is optimal. We review the elements of this definition one by one.

The closure and the homotopies. The necessity of the closure is nicely demonstrated by Figure 1, and needs no further motivation. The homotopy is the natural context for a discussion of end rotation, but the aim of such a discussion is to arrive at an expression which, in accordance with our intuition, depends only on the initial and final states. We achieve this aim only partially: it is true that expression (12) is a function of the initial and final conformations of the open rod alone, but this expression is only valid under certain conditions on the homotopy. The question therefore remains to what extent the restrictions on the homotopy are necessary.

The planarity of the closure. For a well-defined open-rod writhe $W r^{\circ}$, some condition on the closure is clearly necessary, since $W r^{o}$ is defined in terms of the writhe of the closure. For the current definition of $\mathcal{A}_{r_{0}}^{1}$, planarity of the closure in only the initial and final conformations is sufficient-but see Conjecture 4.1 below for an alternative.

The non-opposition condition. The non-opposition condition listed in the definition of $\mathcal{A}_{r_{0}}^{1}$ (condition 5) is imposed by the application of Theorem 2.5. This might seem to be merely a technical restriction: after all, the open writhe is defined in terms of the writhe of the closed curve (Definition 2.3), and the latter is well-defined even if, somewhere along the homotopy, the non-opposition condition is violated. Therefore it might be expected that the statement of well-posedness holds true without condition 5 (even though our proof evidently does not), and that only non-self-intersection of the closed structure is required. 
In fact the situation is not that simple. Figure 3 shows homotopy paths connecting the reference conformation (a) with the deformed rod-closure combinations (b), (c) and (d), where the rod itself (represented by the thick line) is the same in each of the three deformed states. We can imagine the deformed rod to be nearly planar, with the two strands crossing at a short distance from each other. Then the rod and its closure in case (b) have writhe close to -11 In case (c) one adds or subtracts 1 to the writhe of the rod-closure combination for each full turn of the end. The writhe of the combination can therefore be made arbitrarily large. In case (d), finally, the writhe is close to -2 .

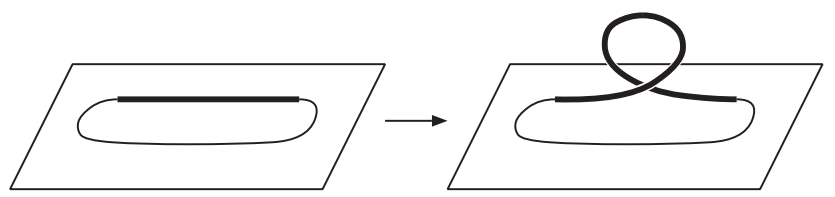

(a)

(b)

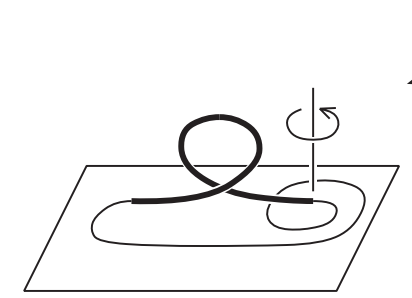

(c)
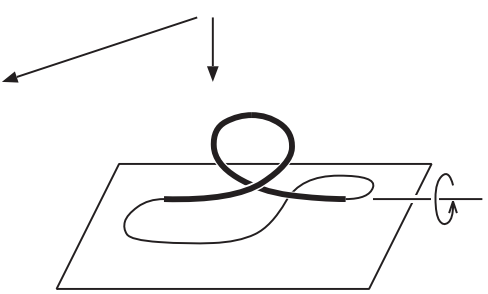

(d)

FIG. 3. An example showing that the non-opposition condition 5 in Definition 2.2 cannot be disposed of. In the three final states (b), (c) and (d), with identical shapes for the open rod, the closed curve has different values of writhe. In going from (b) to either (c) or (d) the non-opposition condition is violated.

It is not difficult to see that one may construct a homotopy from (a) to (b) without violating the non-opposition condition, provided the loop has been twisted through an angle strictly less than $\pi$. Since the concatenation homotopies (a) $\rightarrow$ (b) $\rightarrow$ (c) and $(\mathrm{a}) \rightarrow$ (b) $\rightarrow$ (d) satisfy all conditions of Definition 2.2 other than the non-opposition condition, it follows from Theorem 2.4 that these homotopies cannot be constructed without violating the non-opposition condition. This may also be verified by inspection.

This example shows that simply removing condition 5 from Definition 2.2 leads to ambiguities in the definition of writhe, implying that different homotopies within this larger class lead to different end rotations. The example also suggests that if a welldefined writhe is to be constructed without imposing the non-opposition condition, then additional restrictions must be imposed on the closure. In homotopy (b) $\rightarrow$ (c) the closure remains planar throughout the homotopy, but the end tangents vary; in homotopy (b) $\rightarrow$ (d) the end tangents are constant, but the closure is only planar at the beginning

\footnotetext{
${ }^{1}$ This may be verified by using the characterisation of writhe as the average of the directional writhing number, as explained in Section 2 This number can be determined by counting signed crossings in a projection of the curve onto a plane.
} 
and the end of the homotopy. We conjecture that ruling out these two cases will result in a well-defined writhe:

Conjecture 4.1. On the set

$$
\widetilde{\mathcal{A}_{r_{0}}^{1}}=\left\{\quad\left(r, d_{1}\right) \in \mathcal{A}^{0} \text { such that } \exists\left(\bar{r}, \bar{d}_{1}\right) \in C^{2}\left([0, M] \times[0,1] ; \mathbb{R}^{3} \times S^{2}\right):\right.
$$

1. for each $\lambda, \bar{r}(\cdot, \lambda)$ is an unknotted, non-self-intersecting closed curve,

2. $|\dot{\bar{r}}(s, \lambda)| \neq 0 \quad$ and $\quad \dot{\bar{r}}(s, \lambda) \cdot \bar{d}_{1}(s, \lambda)=0 \quad$ for $s \in[0, M], \lambda \in\{0,1\}$,

3. $\left(\bar{r}, \bar{d}_{1}\right)(s, 1)=\left(r, d_{1}\right)(s)$ for $s \in[0, L]$,

4. $\bar{r}(s, 0)=r_{0}(s)$ for $s \in[0, M]$,

$6^{\prime} .\{\bar{r}(s, \lambda): s \in[L, M]\}$ is a planar curve for all $\lambda \in[0,1]$,

7. $\partial_{\lambda} \dot{\bar{r}}(0, \lambda)=\partial_{\lambda} \dot{\bar{r}}(L, \lambda)=0$ for all $\left.\lambda \in[0,1]\right\}$,

the open writhe given by (9) is a function of $\left(r, d_{1}\right)$ alone.

The reference curve. Deformation histories require a reference conformation to start from (or to end in). The class $\mathcal{A}_{r_{0}}^{1}$ is defined for a fixed closed reference curve $r_{0}$ (which may or may not include the unstressed centerline of the rod). The open writhe $W r^{o}$ will, in general, depend on the choice of this curve; on the one hand, by the fact that the reference curve restricts the class of admissible homotopies via the non-opposition condition, and on the other hand, by the explicit dependence on $t_{0}$ in (12). Similarly, the end rotation $R$ will depend on $r_{0}$, as is to be expected since $R$ is defined (in Definition 2.11) as the end rotation incurred in deforming $\left.\left(r_{0}, \bar{d}_{1}(\cdot, 0)\right)\right|_{[0, L]}$ into $\left(r, d_{1}\right)$.

For certain cases, however, the dependence can be described more precisely, as in Theorem 2.9 for straight reference curves (in 13 a similar result is proved under rotation of more general reference curves). For instance, one could extend Definition [2.11, Lemma 2.12 and Corollary 2.13 to a larger class than $A_{r_{0}}^{2}$ by requiring of $\left.r_{0}\right|_{[0, L]}$ only that its end tangents be equal. A complication would arise, however, in that violation of the non-opposition condition no longer coincides with the Euler-angle singularity. Consequently, the non-opposition condition would no longer assure us of $C^{1}$-regularity of $\phi$, $\psi$, and $\theta$.

We now briefly discuss two other interesting aspects of the approach outlined above.

Euler-angle singularity vs. the non-opposition condition. When the reference conformation is straight and end tangents remain constant during the homotopy, the nonopposition condition is equivalent to avoidance of the Euler-angle singularity at $\theta=\pi$. It should not be concluded from this, however, that the two are one and the same thing, although both have their origin in the topology of $S^{2}$.

The Euler-angle singularity results from the fact that $S^{2}$ is not homeomorphic to (any part of) $\mathbb{R}^{2}$. Any parametrisation of $S^{2}$ by a single cartesian coordinate system will therefore have at least one singular point. On the other hand, the non-opposition condition is necessary - in this paper - for the single-integral representation of writhe in Theorem 2.5. In this representation the ambiguity of area 'enclosed' by a curve on $S^{2}$ is resolved by taking a perturbation approach. The non-opposition condition reflects the unavoidable limits of this approach. 
Open writhe is not rotation invariant. The definition of $\mathcal{A}_{r_{0}}^{1}$ depends on the choice of the reference conformation. For a given reference conformation, an open rod in $\mathcal{A}_{r_{0}}^{1}$ may not be freely rotated without leaving $\mathcal{A}_{r_{0}}^{1}$. This is readily demonstrated by rotating the reference conformation itself: after a rotation through $\pi$ about an axis perpendicular to the plane of the reference curve, the non-opposition condition is violated at every point on the curve.

This may lead to surprising results. In Figure 4 two homotopies are shown. The first is a variation on homotopy (a) $\rightarrow$ (b) of Figure 3, while in the second we lengthen the open-rod part and shorten the closure part. In addition, we construct the homotopies such that the final conformations are close, up to a rotation (emphasized by the mark at one end of the open rod). In (a) the open writhe is close to 1 , while in (b) it is close to 0 .

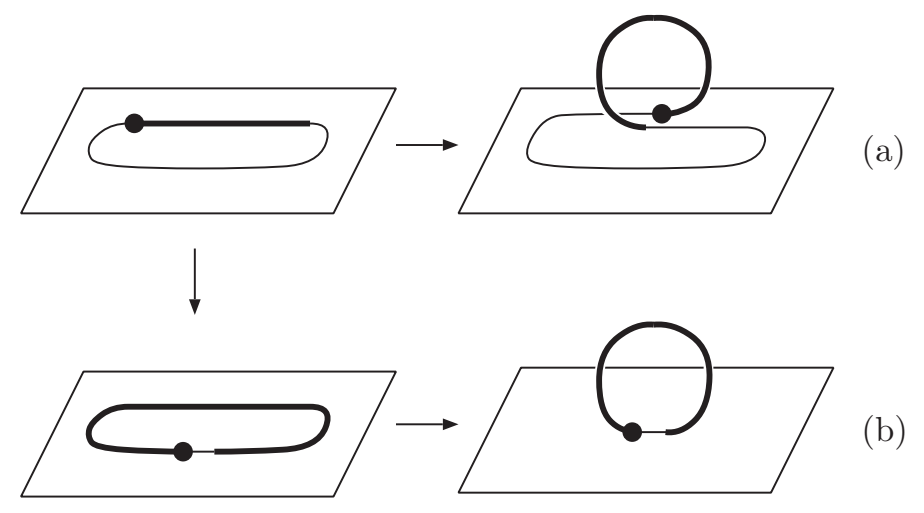

Fig. 4. Two elements of $\mathcal{A}_{r_{0}}^{1}$ that differ only by a rotation, but for which the writhe is different. The dot emphasizes the difference in orientation.

This remark also resolves an issue raised in Remark 2.6 does the open writhe change continuously into the classical writhe for closed rods, when an open rod is transformed into a closed rod by lining up and connecting the ends? The answer is no, as is illustrated by the two homotopies of Figure 4, which lead to the same closed curve with different open-writhe limits; this shows that in the open-to-closed limit the open writhe is not well-defined.

5. Other work. Our aim in this paper is to formulate conditions under which we may infer the value of the end rotation from knowledge of the current (and reference) position of the rod; in the previous sections an example of such a set of conditions is presented and studied. In this section we will briefly discuss how other authors have dealt with the issue of a non-conservative end rotation, and compare their approaches to ours.

The first category of authors consists of those who simply use formulae such as (17), often while noting the difficulties and assumptions. Our treatment of Section 3 is of this type. With the counterexamples of Section 4 in mind this approach needs no further discussion. 
Closures of one type or other appear in various works. Marko [11] defines a closure for open rods with clamped ends by extending two straight lines out to infinity from the rod ends, a type of closure that is essentially indistinguishable from that of Alexander \& Antman [2]. Rossetto \& Maggs [14] extend this concept to open rods with free ends but with equal end tangents, and comment on the relation with a closure that becomes arbitrarily large. Closures of this type, straight lines to infinity, are an interesting alternative to our approach, and we feel they should be investigated further.

Rossetto \& Maggs [14 also take a different position when it comes to determining end rotation practically. They discuss the possibility in today's routine single-DNAmolecule experiments [16] of closure crossings (the molecules may loop around their magnetically supported ends in the manner illustrated in Figure 10, but, adopting a statistical point of view, then simply choose to disregard such closure crossings, arguing that these violations of link conservation are scarce on the time scale that they consider. Note that such an assumption is only possible under non-stationary conditions, i.e., when considering finite-time ensembles; any positive probability of closure crossing, regardless of how small, eliminates the distinction between link classes, and therefore alters all (infinite-time) equilibrium distributions.

In order to avoid the awkward non-opposition condition in Fuller's second theorem there have been direct approaches, based on a closure and calculation of the double integral (5) instead. In [15, 12 simple shapes are considered with planar closures for which the integral can be evaluated explicitly. It is then shown that the closure gives a relative contribution to the writhe which tends to zero as the length of the rod tends to infinity. The double integral is also used in the numerical study in [19, where it is shown that the contribution to $W r$ from the interaction of the closure with the basic chain is of the order of $1 \%$. Various numerical schemes for the computation of the writhe double integral for a discretised curve are discussed and compared in 8. Useful rigorous error bounds on numerically computed values of $W r$ based on polygonal (i.e., piece-wise linear) approximation are given in [5].

Various generalisations exist of writhe to open curves, without introducing a closure. Some authors have used the identification of writhe with area on $S^{2}$ to generalise the writhe to curves that are open but for which the tantrix traces a closed curve on $S^{2}[10$. While this generalisation provides a well-defined generalised writhe, the lack of a closure implies that there is no equivalent of Theorem 2.1, and therefore the connection between the writhe and the rotation of the ends is lost. Therefore such a generalisation of the writhe, although interesting, does not provide a solution to the problem of this paper 2 The same difficulty holds for the suggestion by Fuller himself [6] of using the property that writhe equals the averaged crossing number as a basis for extension to non-closed curves.

\footnotetext{
${ }^{2}$ Actually, the generalisation suggested in 10 even extends to arbitrary curves by an appeal to the concept of geometric phase [3]; in the context of tantrix curves on $S^{2}$, this amounts to closing the tantrix by part of a great circle [10]. This further generalisation is, however, just as useless for our present purposes, for the same reason.
} 
Appendix A. Proof of Theorem 2.9. Let $f: S^{2} \rightarrow \mathbb{R}$ be the function mentioned in the assertion. Pick $v_{0} \in \Omega$ and let $\Omega_{0}$ be the connected component of $\Omega$ containing $v_{0}$. Define the set

$$
A=\left\{v \in \Omega_{0}: f(v)=f\left(v_{0}\right)\right\} .
$$

The function $f$ is continuous on $\Omega_{0}$, implying that the set $A$ is relatively closed in $\Omega_{0}$. We will show below that $f$ is constant on all open balls $B \subset \Omega_{0}$, implying that $A$ is also open. Since $A$ is non-empty it follows that $A=\Omega_{0}$ and the lemma is proved.

For a given vector $\omega \in S^{2}$, let $R_{\phi}$ denote the rotation about $\omega$ through an angle $\phi$. We fix the direction of rotation in the following way: with respect to an orthonormal basis $(\omega, w, \omega \times w)$ for a suitable $w \in S^{2}$, write $R_{\phi}$ as

$$
R_{\phi}=\left(\begin{array}{ccc}
1 & 0 & 0 \\
0 & \cos \phi & -\sin \phi \\
0 & \sin \phi & \cos \phi
\end{array}\right) .
$$

With this choice,

$$
\left.\frac{d}{d \phi} R_{\phi} v\right|_{\phi=0}=\omega \times v \quad \text { for any } v \in S^{2} .
$$

Set $v_{\phi}=R_{\phi} v$. Using equation (25), we have

$$
\frac{d}{d \phi} \frac{v_{\phi} \times t}{1+v_{\phi} \cdot t} \cdot \dot{t}=\frac{\left[\left(1+v_{\phi} \cdot t\right)\left(\left(\omega \times v_{\phi}\right) \times t\right)-\left(v_{\phi} \times t\right)\left(\left(\omega \times v_{\phi}\right) \cdot t\right)\right]}{\left(1+v_{\phi} \cdot t\right)^{2}} \cdot \dot{t} .
$$

Setting $\gamma=\left|\omega \times v_{\phi}\right|$ we introduce an orthonormal coordinate system

$$
e_{1}=v_{\phi}, \quad e_{2}=\gamma^{-1} \omega \times v_{\phi}, \quad e_{3}=\gamma^{-1} v_{\phi} \times\left(\omega \times v_{\phi}\right),
$$

and we write $t_{1}, t_{2}, t_{3}$ for the coordinates of $t$ with respect to this basis; these are functions of the curve parameter $s$. The right-hand side of (26) becomes

$$
\gamma \frac{\left(1+t_{1}\right)\left(t_{3} \dot{t}_{1}-t_{1} \dot{t}_{3}\right)-t_{2}\left(t_{2} \dot{t}_{3}-t_{3} \dot{t}_{2}\right)}{\left(1+t_{1}\right)^{2}} .
$$

Using the equalities $t_{1}^{2}+t_{2}^{2}+t_{3}^{2}=1$ and $t_{1} \dot{t}_{1}+t_{2} \dot{t}_{2}+t_{3} \dot{t}_{3}=0$ this is seen to be equal to

$$
-\gamma \frac{d}{d s} \frac{t_{3}}{1+t_{1}}
$$

Therefore

$$
\frac{d}{d \phi} \int \frac{v_{\phi} \times t}{1+v_{\phi} \cdot t} \cdot \dot{t} d s=-\gamma \int \frac{d}{d s} \frac{t_{3}}{1+t_{1}} d s=0 .
$$

The last equality results from the assumption of aligned end tangents. This proves the theorem.

\section{REFERENCES}

[1] J. Aldinger, I. Klapper, and M. Tabor. Formulae for the calculation and estimation of writhe. $J$. Knot Theory and its Ramifications, 4(3):343-372, 1995. MR.1347359 (97d:57003)

[2] J.C. Alexander and S.S. Antman. The ambiguous twist of Love. Quarterly of Applied Math., 40:8392, 1982. MR652052 (84c:73052)

[3] M. V. Berry. Quantal phase factors accompanying adiabatic changes. Proc. R. Soc. Lond. A, 392:4557, 1984. MR738926 (85i:81022)

[4] G. Călugăreanu. Sur les classes d'isotopie des nœuds tridimensionnels et leurs invariants. Czechoslovak Math. J., 11:588-625, 1961. MR0149378(26:6868) 
[5] J. Cantarella. On comparing the writhe of a smooth curve to the writhe of an inscribed polygon. SIAM J. Numer. Anal., 42:1846-1861, 2005. MR2139226 (2006e:53005)

[6] F.B. Fuller. The writhing number of a space curve. Proc. Nat. Acad. Sciences USA, 68(4):815-819, 1971. MR0278197(43:3928)

[7] F.B. Fuller. Decomposition of the linking number of a closed ribbon: A problem from molecular biology. Proc. Nat. Acad. Sciences USA, 75:3557-3561, 1978. MR0490004(58:9367)

[8] K. Klenin and J. Langowski. Computation of writhe in modeling of supercoiled DNA. Biopolymers, 54:307-317, 2000.

[9] A.E.H. Love. A Treatise on the Mathematical Theory of Elasticity. Dover Publications, 4th edition, 1944. MR0010851(6:79e)

[10] A.C. Maggs. Twist and writhe dynamics of stiff polymers. Phys. Rev. Lett., 85:5472-5475, 2000.

[11] J.F. Marko. Supercoiled and braided DNA under tension. Phys. Rev. E, 55(2):1758-1772, 1997.

[12] S. Neukirch and G.H.M. van der Heijden. Geometry and mechanics of uniform $n$-plies: from engineering ropes to biological filaments. J. Elasticity, 69:41-72, 2002. MR2020510(2004i:74070)

[13] R. Planqué. Constraints in Applied Mathematics: Rods, Membranes, and Cuckoos. Ph.D. thesis, Technische Universiteit Eindhoven, 2005.

[14] V. Rossetto and A. C. Maggs. Writhing geometry of open DNA. J. Chem. Phys., 118:9864-9874, 2003.

[15] E.L. Starostin. On the writhe of non-closed curves. arXiv:physics/0212095, 2002.

[16] T.R. Strick, J.F. Allemand, D. Bensimon, A. Bensimon, and V. Croquette. The elasticity of a single supercoiled DNA molecule. Science, 271, 1835-1837 1996.

[17] G.H.M. van der Heijden. The static deformation of a twisted elastic rod constrained to lie on a cylinder. Proc. Roy. Soc. London A, 457:695-715, 2001. MR1841581 (2002d:74040)

[18] G.H.M. van der Heijden, M.A. Peletier, and R. Planqué. Self-contact for rods on cylinders. Arch. Rat. Mech. Anal., 182:471-511, 2006.

[19] A.V. Vologodskii and J.F. Marko. Extension of torsionally stressed DNA by external force. Biophys. J., 73:123-132, 1997.

[20] J.H. White. Self-linking and the Gauss integral in higher dimensions. Amer. J. Math., 91:693-728, 1969. MR 0253264(40:6479) 